\title{
Imaginary potential of moving quarkonia in a D-instanton background
}

\author{
Zi-qiang Zhang,, , * De-fu Hou, ${ }^{2, \text {, }}$ and Gang Chen ${ }^{1}$, 田 \\ ${ }^{1}$ School of mathematics and physics, China University of Geosciences(Wuhan), Wuhan 430074, China \\ ${ }^{2}$ Key Laboratory of Quark and Lepton Physics (MOE), \\ Central China Normal University, Wuhan 430079, China
}

\begin{abstract}
The imaginary part of the inter-quarks potential of moving heavy quarkonia is investigated in a dual supergravity of the AdS background deformed by dilaton, which induces the gauge field condensate in the dual gauge theory. We analyze the quark anti-quark pair moving transverse and parallel to the plasma wind, respectively. It is shown that for both cases the presence of D-instanton density tends to increase the inter-distance and decrease the imaginary potential, reverse to the effect of the pair's velocity. Moreover, it is found that the D-instanton density has stronger effects for the parallel case rather than transverse.
\end{abstract}

PACS numbers: 12.38.Mh, 11.25.Tq, 11.15.Tk

\section{INTRODUCTION}

The experimental program at RHIC and LHC have produced a new state of matter so-called quark gluon plasma (QGP) [1 3]. One important experimental signal for QGP formation is melting of quarkonia. It was suggested earlier that the main mechanism responsible for this suppression is color screening [4]. But recently some authors argued that the imaginary part of the potential, $\mathrm{ImV}_{Q Q}$, can yield this suppression as well [5] 8 ]. In addition, this quantity can be used to estimate the thermal width of quarkonia. In the framework of weakly coupled theories, $\operatorname{Im} \mathrm{V}_{Q Q}$ has been studied in many works, see e.g. [9 12]. As we know, at very high temperatures (and/or densities), QCD may be treated perturbatively, for instance, perturbative calculations taking into account hard thermal loops are actually able to reproduce lattice QCD results for the equation of state for temperatures above T $\sim 300 \mathrm{MeV}$ (see. e.g. [13]). However, at lower temperatures, for example, around the QCD crossover region, T $155 \mathrm{MeV}$, the perturbative calculation may not be fully trusted, implying that nonperturbative methods are required in this case. On the other hand, much experiment data indicates that QGP is strongly coupled and behaves like a nearly idea fluid [3, 14, 15]. Therefore, it would be interesting to study the $\operatorname{ImV}_{Q Q}$ via the use of non-petubative methods, such as the AdS/CFT correspondence [16 18].

AdS/CFT, the duality between a string theory in AdS space and a conformal field theory in the physical space-time, has yielded many important insights for studying different aspects of QGP [19]. In this approach, J. Noronha and A. Dumitru have studied the imaginary potential and thermal width of quarkonia for $\mathcal{N}=4$ SYM theory in their seminal work [20]. Therein, the $\operatorname{ImV}_{Q Q}$ is related to the effect of thermal fluctuations due to the interactions between the heavy quarks and the medium. Later, this idea has been extended to various cases. For example, the $\operatorname{ImV} V_{Q Q}$ of static quarkonia is investigated in [21, 22]. The $\operatorname{ImV}_{Q Q}$ of moving quarkonia has been analyzed in 23, 24]. The finite 't Hooft coupling corrections on $\operatorname{ImV}_{Q Q}$ is discussed in [25]. The influence of chemical potential on $\operatorname{ImV} V_{Q Q}$ has been addressed in [26]. The study of $\operatorname{ImV}_{Q Q}$ in some AdS/QCD models, can be found, for example, in [27, 28]. Also, there are other ways to study $\operatorname{ImV}_{Q Q}$ from AdS/CFT, see [29, 30].

Now we would like to give such analysis in a D3/D-instanton background, which corresponds to the Yang-Mills theory in the deconfining, high-temperature phase. The background geometry contains a nontrivial dilaton giving a nonzero gluon condensation $q \propto<\operatorname{Tr} F^{\mu \nu} F_{\mu \nu}>$, where $q$ is also regarded as the D-instanton density 31. It was argued [32, 33] that the features of the D3/D-instanton geometry are similar to QCD at finite temperature. Thus, one expects that the results obtained from this theory should shed qualitative insights into analogous questions in QCD. Due to this characteristic, many quantities have been studied in the D-instanton background, such as phase transitions [32], flavor quark [33], heavy quark potential and jet quenching parameter [34].

In this paper, we will investigate the imaginary potential of moving quarkonia in the D-instanton background. More specifically, we would like to see how the D-density affects $\operatorname{ImV}_{Q Q}$ in this case. Also, it would be interesting to compare the effects of the D-density with those of the velocity. These are the main motivations of the present work.

\footnotetext{
*Electronic address: zhangzq@cug.edu.cn

$\dagger$ Electronic address: houdf@mail.ccnu.edu.cn

${ }^{\ddagger}$ Electronic address: chengang1@cug.edu.cn
} 
The outline of the paper is as follows. In the next section, we briefly review the geometry of the D-instanton background at finite temperature. In section 3, we study the imaginary potential for the pair moving transverse and parallel to the plasma wind one by one. The last part is devoted to conclusion and discussion.

\section{BACKGROUND GEOMETRY}

Let us begin with a briefly review of the D-instanton background. The geometry is a finite temperature extension of D3/D-instanton background given in [33]. The background has a five-form field strength and an axion field which couples to D3 and D-instanton, respectively. In Einstein frame the ten dimensional super-gravity action is given by [35, 36]

$$
S=\frac{1}{\kappa} \int d^{10} x \sqrt{g}\left(R-\frac{1}{2}(\partial \Phi)^{2}+\frac{1}{2} e^{2 \Phi}(\partial \chi)^{2}-\frac{1}{6} F_{(5)}^{2}\right),
$$

where $\Phi$ is the dilaton, $\chi$ stands for the axion. By setting $\chi=-e^{-\Phi}+\chi_{0}$, the dilaton term and the axion term can cancel. Then the solution of (11) can be written as [37]

$$
d s^{2}=e^{\frac{\Phi}{2}}\left[-\frac{r^{2}}{R^{2}} f(r) d t^{2}+\frac{r^{2}}{R^{2}} d \vec{x}^{2}+\frac{1}{f(r)} \frac{R^{2}}{r^{2}} d r^{2}\right]
$$

with

$$
e^{\Phi}=1+\frac{q}{r_{t}^{4}} \log \frac{1}{f(r)}, \quad f(r)=1-\frac{r_{t}^{4}}{r^{4}},
$$

where $R$ is the AdS radius with $R^{4}=4 \pi g_{s} N_{c} \alpha^{\prime 2} . \vec{x}=x_{1}, x_{2}, x_{3}$ are the boundary coordinates. $r$ stands for the radial coordinate. The event horizon and the boundary are located at $r=r_{t}$ and $r=\infty$, respectively. The Hawking temperature is $T=r_{t} /\left(\pi R^{2}\right)$. The parameter $q$ refers to the D-instanton density as well as the vacuum expectation value of the gauge field condensate. Note that for $q=0$ in (2) the $A d S_{5}$-Schwarzschild metric is reproduced.

The next step is to make the pair moving. Supposing that the plasma is at rest and the frame is moving in one direction, i.e., the $x_{3}$ direction, so that

$$
d t=d t^{\prime} \cosh \beta-d x_{3}^{\prime} \sinh \beta, \quad d x_{3}=-d t^{\prime} \sinh \beta+d x_{3}^{\prime} \cosh \beta,
$$

where $\beta$ is called the velocity or rapidity.

Inserting (44) into (2) and dropping the primes, one obtains the boosted metric as

$$
\begin{aligned}
d s^{2}=e^{\frac{\Phi}{2}} & \left(-\frac{r^{2}}{R^{2}} f(r) \cosh ^{2} \beta+\frac{r^{2}}{R^{2}} \sinh ^{2} \beta\right) d t^{2}-2 \sinh \beta \cosh \beta\left(\frac{r^{2}}{R^{2}}-\frac{r^{2}}{R^{2}} f(r)\right) d t d x_{3} \\
& \left.+\left(-\frac{r^{2}}{R^{2}} f(r) \sinh ^{2} \beta+\frac{r^{2}}{R^{2}} \cosh ^{2} \beta\right) d x_{3}^{2}+\frac{r^{2}}{R^{2}}\left(d x_{1}^{2}+d x_{2}^{2}\right)+\frac{R^{2}}{r^{2}} f(r)^{-1} d r^{2}\right] .
\end{aligned}
$$

Noticed that for $\beta=0$ in (5) the metric of (2) is recovered.

\section{IMAGINARY POTENTIAL}

We now follow the argument in [20] to investigate the imaginary potential of a moving quark anti-quark pair for the background metric (5). In general, to analyze the moving case, one needs to consider different alignments for the pair with respect to the plasma wind, i.e., transverse $(\theta=\pi / 2)$, parallel $(\theta=0)$, or arbitrary direction $(\theta)$. In this work, we discuss two extreme cases: $\theta=\pi / 2$ and $\theta=0$.

\section{A. Transverse to the wind $(\theta=\pi / 2)$}

In this subsection, we consider the system perpendicularly to the wind in the $x_{1}$ direction, the coordinates are parameterized by

$$
t=\tau, \quad x_{1}=\sigma, \quad x_{2}=0, \quad x_{3}=0, \quad r=r(\sigma)
$$


where the quark and anti-quark are located at $x_{1}=-\frac{L}{2}$ and $x_{1}=\frac{L}{2}$ with $L$ the inter-distance.

The string action is

$$
S=-\frac{1}{2 \pi \alpha^{\prime}} \int d \tau d \sigma \mathcal{L}=-\frac{1}{2 \pi \alpha^{\prime}} \int d \tau d \sigma \sqrt{-g},
$$

where $g$ is the determinant of the induced metric with

$$
g_{\alpha \beta}=g_{\mu \nu} \frac{\partial X^{\mu}}{\partial \sigma^{\alpha}} \frac{\partial X^{\nu}}{\partial \sigma^{\beta}}
$$

with $g_{\mu \nu}$ the metric and $X^{\mu}$ the target space coordinates.

Substituting (6) into (5), one obtains the induced metric as

$$
g_{00}=e^{\frac{\Phi}{2}}\left[\frac{r^{2}}{R^{2}} f(r) \cosh ^{2} \beta-\frac{r^{2}}{R^{2}} \sinh ^{2} \beta\right], \quad g_{11}=e^{\frac{\Phi}{2}}\left[\frac{r^{2}}{R^{2}}+\frac{R^{2}}{f(r) r^{2}} \dot{r}^{2}\right],
$$

with $\dot{r}=d r / d \sigma$.

Then the corresponding lagrangian density can be written as

$$
\mathcal{L}=\sqrt{a(r)+b(r) \dot{r}^{2}}
$$

with

$$
\begin{aligned}
& a(r)=e^{\Phi}\left[\frac{r^{4}}{R^{4}} f(r) \cosh ^{2} \beta-\frac{r^{4}}{R^{4}} \sinh ^{2} \beta\right], \\
& b(r)=e^{\Phi}\left[\cosh ^{2} \beta-\frac{1}{f(r)} \sinh ^{2} \beta\right] .
\end{aligned}
$$

Note that the action does not depend on $\sigma$ explicitly, so the Hamiltonian is a constant,

$$
\mathcal{L}-\frac{\partial \mathcal{L}}{\partial \dot{r}} \dot{r}=\text { constant }
$$

Considering the boundary condition at $\sigma=0$,

$$
\dot{r}=0, \quad r=r_{c},
$$

one finds

$$
\dot{r}=\frac{d r}{d \sigma}=\sqrt{\frac{a^{2}(r)-a(r) a\left(r_{c}\right)}{a\left(r_{c}\right) b(r)}}
$$

with

$$
\begin{aligned}
& a\left(r_{c}\right)=e^{\Phi\left(r_{c}\right)}\left[\frac{r_{c}^{4}}{R^{4}} f\left(r_{c}\right) \cosh ^{2} \beta-\frac{r_{c}^{4}}{R^{4}} \sinh ^{2} \beta\right], \\
& f\left(r_{c}\right)=1-\frac{r_{t}^{4}}{r_{c}^{4}}, \quad e^{\Phi\left(r_{c}\right)}=1+\frac{q}{r_{t}^{4}} \log \frac{1}{f\left(r_{c}\right)},
\end{aligned}
$$

where $r=r_{c}$ is the deepest point of the U-shaped string.

Integrating (14), the inter-distance of $Q \bar{Q}$ reads

$$
L=2 \int_{r_{c}}^{\infty} d r \sqrt{\frac{a\left(r_{c}\right) b(r)}{a^{2}(r)-a(r) a\left(r_{c}\right)}} .
$$

Substituting (14) into (7) and subtracting the self energy of the two quarks [38 40], the real part of the heavy quark potential is obtained as

$$
\operatorname{ReV}_{Q \bar{Q}}=\frac{1}{\pi \alpha^{\prime}} \int_{r_{c}}^{\infty} d r\left[\sqrt{\frac{a(r) b(r)}{a(r)-a\left(r_{c}\right)}}-\sqrt{b\left(r_{0}\right)}\right]-\frac{1}{\pi \alpha^{\prime}} \int_{r_{t}}^{r_{c}} d r \sqrt{b\left(r_{0}\right)}
$$


with $b\left(r_{0}\right)=b(r \rightarrow \infty)$.

On the other hand, using the thermal worldsheet fluctuation method [20], the imaginary part of the potential is found to be

$$
\operatorname{Im} V_{Q \bar{Q}}=-\frac{1}{2 \sqrt{2} \alpha^{\prime}}\left[\frac{a^{\prime}\left(r_{c}\right)}{2 a^{\prime \prime}\left(r_{c}\right)}-\frac{a\left(r_{c}\right)}{a^{\prime}\left(r_{c}\right)}\right] \sqrt{b\left(r_{c}\right)},
$$

with

$$
\begin{gathered}
a^{\prime}\left(r_{c}\right)=e^{\Phi^{\prime}\left(r_{c}\right)} r_{c}^{4}\left[f\left(r_{c}\right) \cosh ^{2} \beta-\sinh ^{2} \beta\right]+e^{\Phi\left(r_{c}\right)}\left[4 r_{c}^{3} f\left(r_{c}\right) \cosh ^{2} \beta+r_{c}^{4} f^{\prime}\left(r_{c}\right) \cosh ^{2} \beta-4 r_{c}^{3} \sinh ^{2} \beta\right], \\
a^{\prime \prime}\left(r_{c}\right)=e^{\Phi^{\prime \prime}\left(r_{c}\right)} r_{c}^{4}\left[f\left(r_{c}\right) \cosh ^{2} \beta-\sinh ^{2} \beta\right]+e^{\Phi^{\prime}\left(r_{c}\right)}\left[8 r_{c}^{3} f\left(r_{c}\right) \cosh ^{2} \beta-8 r_{c}^{3} \sinh ^{2} \beta+2 r_{c}^{4} f^{\prime}\left(r_{c}\right) \cosh ^{2} \beta\right] \\
+e^{\Phi\left(r_{c}\right)}\left[12 r_{c}^{2} f\left(r_{c}\right) \cosh ^{2} \beta+8 r_{c}^{3} f^{\prime}\left(r_{c}\right) \cosh ^{2} \beta+r_{c}^{4} f^{\prime \prime}\left(r_{c}\right) \cosh ^{2} \beta-12 r_{c}^{2} \sinh ^{2} \beta\right], \\
b\left(r_{c}\right)=e^{\Phi\left(r_{c}\right)}\left[\cosh ^{2} \beta-\frac{1}{f\left(r_{c}\right)} \sinh ^{2} \beta\right], \\
e^{\Phi^{\prime}\left(r_{c}\right)}=-q r_{t}^{-4} \frac{f^{\prime}\left(r_{c}\right)}{f\left(r_{c}\right) \log 10}, \quad e^{\Phi^{\prime \prime}\left(r_{c}\right)}=-q r_{t}^{-4} \frac{f^{\prime \prime}\left(r_{c}\right) f\left(r_{c}\right)-f^{\prime}\left(r_{c}\right) f^{\prime}\left(r_{c}\right)}{f^{2}\left(r_{c}\right) \log 10}, \\
f^{\prime}\left(r_{c}\right)=4 r_{t}^{4} r_{c}^{-5}, \quad f^{\prime \prime}\left(r_{c}\right)=-20 r_{t}^{4} r_{c}^{-6},
\end{gathered}
$$

where, for simplicity, we have taken $R=1$. One can check that for $q=0$ in [19) the result of [24] is recovered. And for $q=\beta=0$ in (19), the formula of [20] is reproduced as well.

Before evaluating the imaginary potential, we should pause here to discuss the regime of applicability of this model. It was argued [20, 23] that there are three restrictions to the formula (19). First, the term $b\left(r_{c}\right)$ needs to be positive, which leads to

$$
\varepsilon<\varepsilon_{\max , 1}=\left(1-\tanh ^{2} \beta\right)^{1 / 4}
$$

with $\varepsilon \equiv r_{t} / r_{c}$.

Second, the imaginary potential should be negative, so

$$
\frac{a^{\prime}\left(r_{c}\right)}{2 a^{\prime \prime}\left(r_{c}\right)}-\frac{a\left(r_{c}\right)}{a^{\prime}\left(r_{c}\right)}>0
$$

which yields

$$
\varepsilon>\varepsilon_{\min }
$$

here the length expression of $\varepsilon_{\min }$ is not shown, but one will see its behavior in fig.2 below.

The third restriction is related to the maximum value of the inter-distance. To address this, we plot $L T$ versus $\varepsilon$ for various cases in fig.1, one can see that in each plot there exists a maximum value of $L T_{\text {max }}$, which corresponds to a critical value of $\varepsilon_{\max , 2}$, and that $L T$ is a increasing function of $\varepsilon$ for $\varepsilon<\varepsilon_{\max , 2}$ but a decreasing one for $\varepsilon>\varepsilon_{\max , 2}$. Actually, for the later case it is necessary to consider highly curved configurations which are not solutions of the Nambu-Goto action [41], so here we are mostly interested in the region of $\varepsilon<\varepsilon_{\max , 2}$. Also, it is interesting to mention that $L T_{\max }$ has been used to define the dissociation length for the moving $Q \bar{Q}$, see e.g. [42, 43]. But in this work, we tend to use it to define the region of applicability of the U-shaped string configuration, as follows from [23].

By combining the three restrictions, we plot $\varepsilon_{\min }, \varepsilon_{\max , 1}, \varepsilon_{\max , 2}$ versus $\beta$ for $q=0.2$ in fig.2. Other cases with different values of $q$ have similar picture. From the figures, one can see that these restrictions lead to a narrow window. Or in other words, the regime of applicability of the formula (19) is $\varepsilon_{\min }<\varepsilon<\varepsilon_{\max , 2}$.

To proceed, we study the effects of the velocity and the D-density on the inter-distance. From fig.1, one sees that at a fixed $q$, increasing $\beta$ the value of $L T_{\max }$ decreases. Namely, the velocity has the effect of decreasing the interdistance, consistently with the findings of [23, 24]. Moreover, comparing the left panel with the right one, one finds that at a fixed $\beta$, increasing $q$ leads to increasing the value of $L T_{\max }$. Therefore, one concludes that the D-density affects the inter-distance in the opposite way of the velocity. 

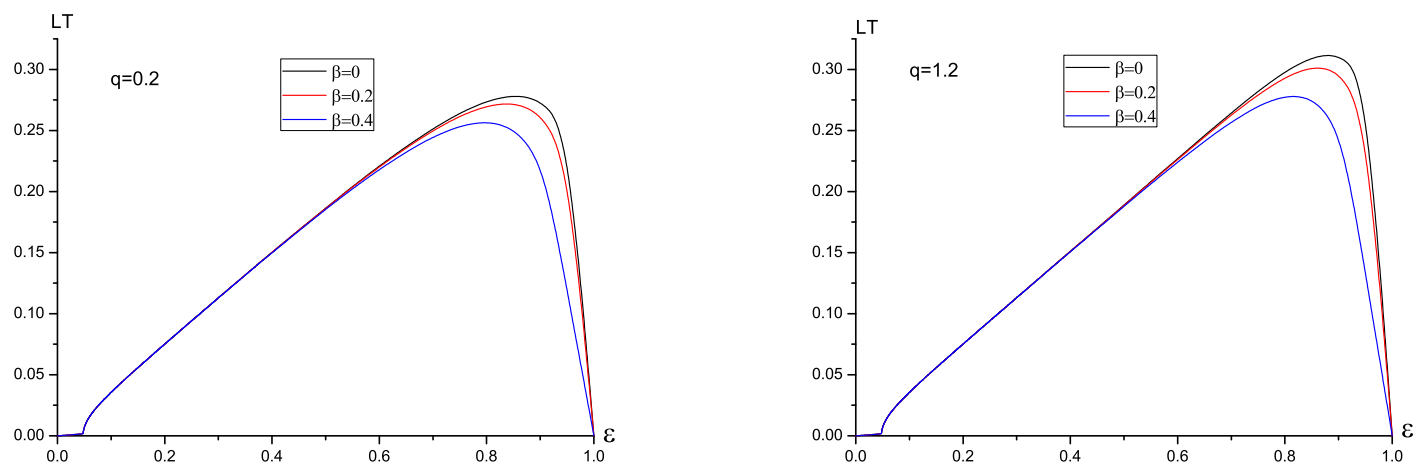

FIG. 1: Plots of $L T$ versus $\varepsilon$ for $\theta=\pi / 2$. Left: $q=0.2$. Right: $q=1.2$. In all of the plots from top to bottom $\beta=0,0.2,0.4$, respectively.

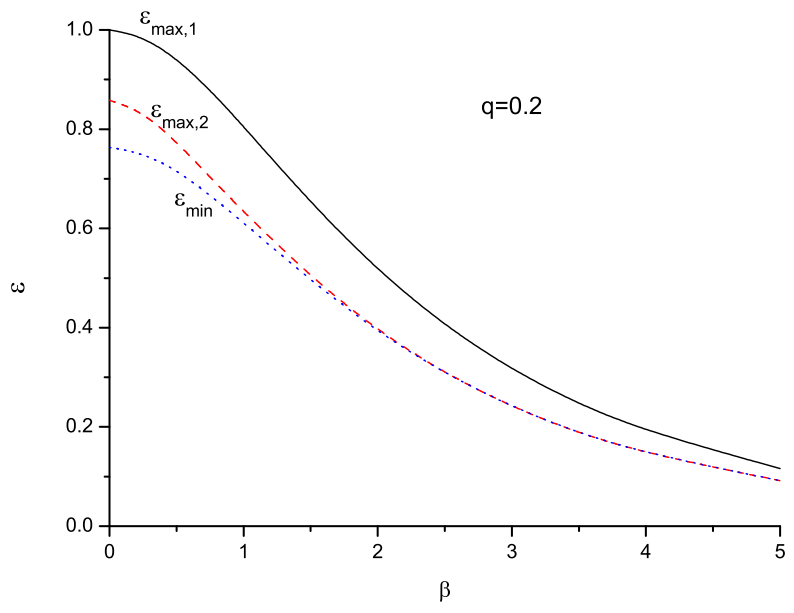

FIG. 2: Limiting curves of $\varepsilon$ versus $\beta$ for $\theta=\pi / 2$ and $q=0.2$. From top to bottom, the three curves represent $\varepsilon_{\max , 1}$ (solid line), $\varepsilon_{\max , 2}\left(\right.$ dash line) and $\varepsilon_{\min }($ dot line), respectively.

Next, we investigate the imaginary potential in the region of $\varepsilon_{\min }<\varepsilon<\varepsilon_{\max , 2}$. In Fig.3, we plot $\operatorname{ImV}_{Q \bar{Q}} /(\sqrt{\lambda} T)$ versus $L T$ for two different values of $q$. The left panel is plotted for $q=0.2$ while the right one is for $q=1.2$. In both panels from left to right $\beta=0.4,0.2,0$, respectively. From the figures, one can see that the imaginary potential starts at a $L_{\min }$, corresponding to $\varepsilon=\varepsilon_{\min }$ or $\operatorname{Im} V_{Q \bar{Q}}=0$, and ends at a $L_{\max }$, corresponding to $\varepsilon_{\max , 2}$. Also, one finds that at a fixed $\beta$, increasing $q$ the imaginary potential decreases. As discussed in 23], the dissociation properties of quarkonia should be sensitive to the imaginary potential, and if the onset of $\operatorname{ImV}_{Q \bar{Q}}$ happens for smaller $L T$, the suppression will be stronger. Thus, we conclude that the presence of the D-instanton density makes the suppression weaker, reverse to the effect of the velocity. Interestingly, it was shown 34 that the D-instanton density has the effect of suppressing the heavy quark potential thus making the quarkonia melt harder, in agreement with the findings here.

\section{B. Parallel to the wind $(\theta=0)$}

In this subsection we discuss the system parallel to the wind. The coordinates are parameterized as

$$
t=\tau, \quad x_{1}=0, \quad x_{2}=0, \quad x_{3}=\sigma, \quad r=r(\sigma),
$$

where the quark and anti-quark are located at $x_{3}=-\frac{L}{2}$ and $x_{3}=\frac{L}{2}$, respectively. 

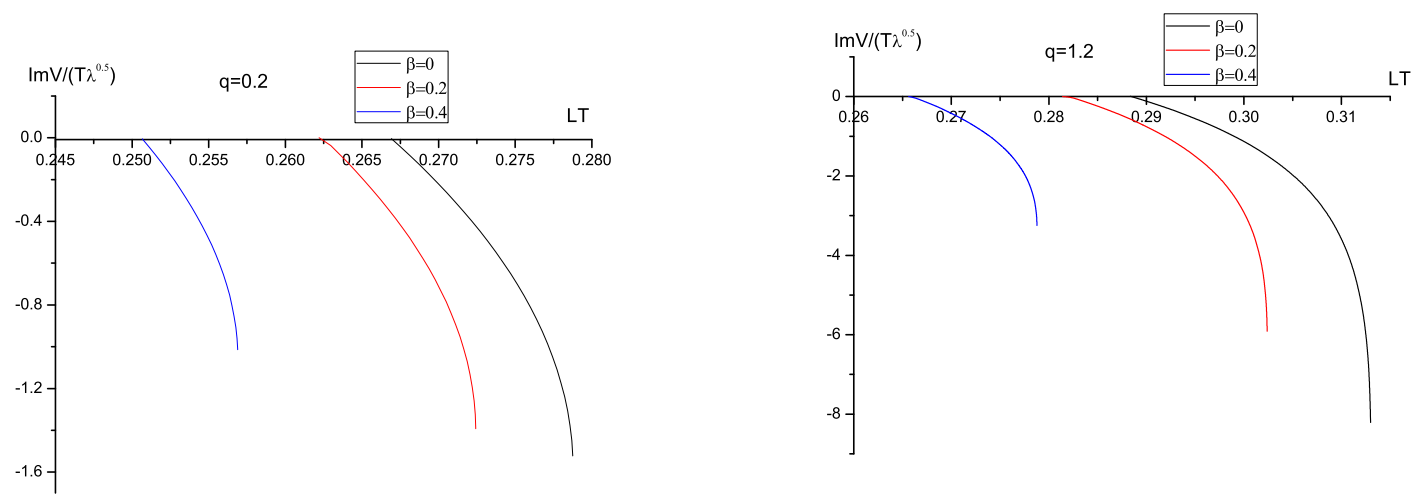

FIG. 3: Plots of $\operatorname{Im} V /(\sqrt{\lambda} T)$ versus $L T$ for $\theta=\pi / 2$. Left: $q=0.2$. Right: $q=1.2$. In all of the plots from left to right $\beta=0.4,0.2,0$, respectively.

The next analysis is similar to the previous subsection, so we just present the final results. The inter-distance is

$$
L=2 \int_{r_{c}}^{\infty} d r \sqrt{\frac{A\left(r_{c}\right) B(r)}{A^{2}(r)-A(r) A\left(r_{c}\right)}},
$$

with

$$
\begin{gathered}
A(r)=e^{\Phi} \frac{r^{4}}{R^{4}}\left[f(r) \sinh ^{4} \beta+f(r) \cosh ^{4} \beta-\sinh ^{2} \beta \cosh ^{2} \beta\left(1+f^{2}(r)\right)\right], \\
A\left(r_{c}\right)=e^{\Phi\left(r_{c}\right)} \frac{r_{c}^{4}}{R^{4}}\left[f\left(r_{c}\right) \sinh ^{4} \beta+f\left(r_{c}\right) \cosh ^{4} \beta-\sinh ^{2} \beta \cosh ^{2} \beta\left(1+f^{2}\left(r_{c}\right)\right)\right], \\
B(r)=e^{\Phi}\left[\cosh ^{2} \beta-\frac{1}{f(r)} \sinh ^{2} \beta\right] .
\end{gathered}
$$

The imaginary potential is

$$
\operatorname{Im} V_{Q \bar{Q}}=-\frac{1}{2 \sqrt{2} \alpha^{\prime}}\left[\frac{A^{\prime}\left(r_{c}\right)}{2 A^{\prime \prime}\left(r_{c}\right)}-\frac{A\left(r_{c}\right)}{A^{\prime}\left(r_{c}\right)}\right] \sqrt{B\left(r_{c}\right)}
$$

with

$$
\begin{aligned}
& A^{\prime}\left(r_{c}\right)=e^{\Phi\left(r_{c}\right)}\left[\left(\sinh ^{4} \beta+\cosh ^{4} \beta\right)\left(4 r_{c}^{3} f\left(r_{c}\right)+r_{c}^{4} f^{\prime}\left(r_{c}\right)\right)-\sinh ^{2} \beta \cosh ^{2} \beta\left(4 r_{c}^{3}+4 r_{c}^{3} f^{3}\left(r_{c}\right)\right.\right. \\
& \left.\left.+2 r_{c}^{4} f\left(r_{c}\right) f^{\prime}\left(r_{c}\right)\right)\right]+e^{\Phi^{\prime}\left(r_{c}\right)} r_{c}^{4}\left[f\left(r_{c}\right) \sinh ^{4} \beta+f\left(r_{c}\right) \cosh ^{4} \beta-\sinh ^{2} \beta \cosh ^{2} \beta\left(1+f^{2}\left(r_{c}\right)\right)\right], \\
& A^{\prime \prime}\left(r_{c}\right)=e^{\Phi^{\prime \prime}\left(r_{c}\right)} r_{c}^{4}\left[f\left(r_{c}\right) \sinh ^{4} \beta+f\left(r_{c}\right) \cosh ^{4} \beta-\sinh ^{2} \beta \cosh ^{2} \beta\left(1+f^{2}\left(r_{c}\right)\right)\right] \\
& +2 e^{\Phi^{\prime}\left(r_{c}\right)}\left[\left(\sinh ^{4} \beta+\cosh ^{4} \beta\right)\left(4 r_{c}^{3} f\left(r_{c}\right)+r_{c}^{4} f^{\prime}\left(r_{c}\right)\right)-\sinh ^{2} \beta \cosh ^{2} \beta\left(4 r_{c}^{3}+4 r_{c}^{3} f^{3}\left(r_{c}\right)+2 r_{c}^{4} f\left(r_{c}\right) f^{\prime}\left(r_{c}\right)\right)\right] \\
& +e^{\Phi}\left(r_{c}\right)\left[\left(\sinh ^{4} \beta+\cosh ^{4} \beta\right)\left(12 r_{c}^{2} f\left(r_{c}\right)+8 r_{c}^{3} f^{\prime}\left(r_{c}\right)+r_{c}^{4} f^{\prime \prime}\left(r_{c}\right)\right)-\sinh ^{2} \beta \cosh ^{2} \beta\left(12 r_{c}^{2}+12 r_{c}^{2} f^{2}\left(r_{c}\right)\right.\right. \\
& \left.\left.+8 r_{c}^{3} f\left(r_{c}\right) f^{\prime}\left(r_{c}\right)+2 r_{c}^{4} f\left(r_{c}\right) f^{\prime \prime}\left(r_{c}\right)+8 r_{c}^{3} f^{\prime}\left(r_{c}\right) f\left(r_{c}\right)+2 r_{c}^{4} f^{\prime}\left(r_{c}\right) f^{\prime}\left(r_{c}\right)\right)\right] \\
& B\left(r_{c}\right)=e^{\Phi\left(r_{c}\right)}\left[\cosh ^{2} \beta-\frac{1}{f\left(r_{c}\right)} \sinh ^{2} \beta\right],
\end{aligned}
$$

and

$$
e^{\Phi^{\prime}\left(r_{c}\right)}=-q r_{t}^{-4} \frac{f^{\prime}\left(r_{c}\right)}{f\left(r_{c}\right) \log 10}, \quad e^{\Phi^{\prime \prime}\left(r_{c}\right)}=-q r_{t}^{-4} \frac{f^{\prime \prime}\left(r_{c}\right) f\left(r_{c}\right)-f^{\prime}\left(r_{c}\right) f^{\prime}\left(r_{c}\right)}{f^{2}\left(r_{c}\right) \log 10}
$$



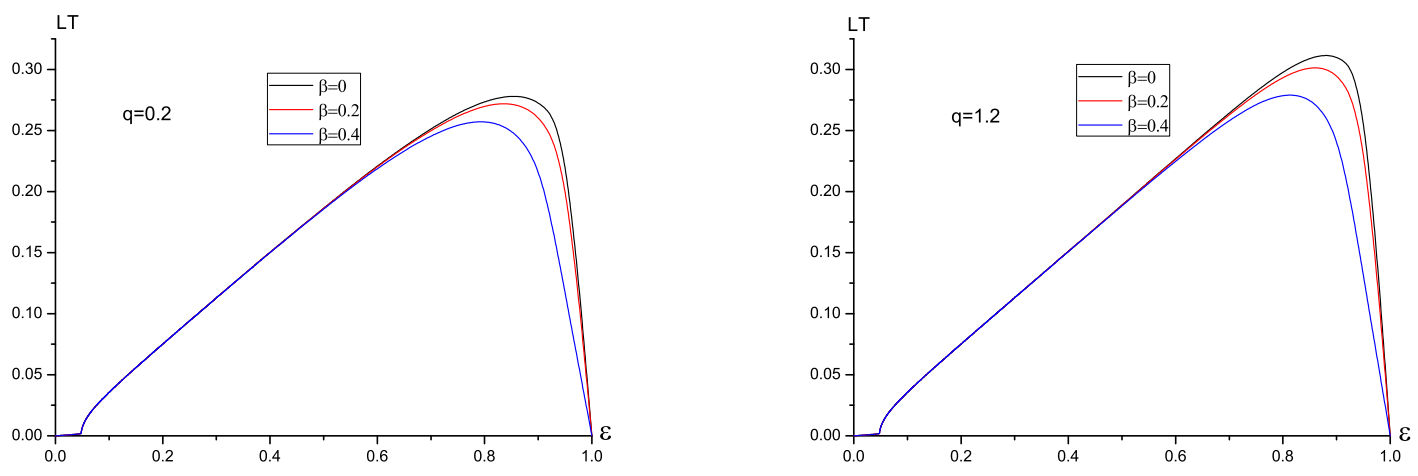

FIG. 4: Plots of $L T$ versus $\varepsilon$ for $\theta=0$. Left: $q=0.2$. Right: $q=1.2$. In all of the plots from top to bottom $\beta=0,0.2,0.4$, respectively.
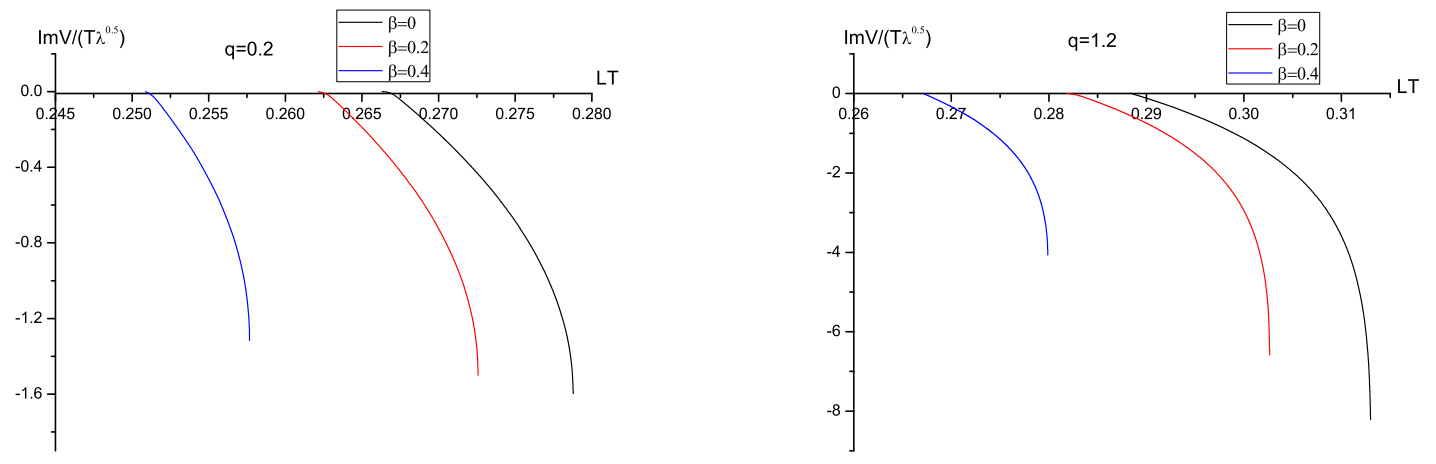

FIG. 5: Plots of $\operatorname{Im} V /(\sqrt{\lambda} T)$ versus $L T$ for $\theta=0$. Left: $q=0.2$. Right: $q=1.2$. In all of the plots from left to right $\beta=0.4,0.2,0$, respectively.

$$
f^{\prime}\left(r_{c}\right)=4 r_{t}^{4} r_{c}^{-5}, \quad f^{\prime \prime}\left(r_{c}\right)=-20 r_{t}^{4} r_{c}^{-6}
$$

Likewise, we plot $L T$ versus $\varepsilon$ and $\operatorname{ImV}_{Q \bar{Q}} /(\sqrt{\lambda} T)$ versus $L T$ for $\theta=0$ in fig.4 and fig.5, respectively. From these figures, one finds that the results are very similar to the transverse case: the D-instanton density increases the inter-distance and decreases the imaginary potential, while the velocity has opposite effects.

Moreover, to compare the effects of the D-instanton density on the imaginary potential between $\theta=0$ and $\theta=\pi / 2$, we plot $\operatorname{ImV}_{Q \bar{Q}} /(\sqrt{\lambda} T)$ versus $L T$ with $\beta=0.4$ for two different $q$ in the left panel of fig.6. The left two adjacent curves correspond to $\operatorname{ImV} V_{Q \bar{Q}, \perp} /(\sqrt{\lambda} T)$ (lower line) and $\operatorname{ImV}_{Q \bar{Q}, \|} /(\sqrt{\lambda} T)$ (upper line) for $q=0.2$ while the right ones correspond to their counterparts for $q=1.2$. One sees that at fixed $\beta$, increasing $q$, the differences between the onsets of the $\operatorname{ImV}_{Q \bar{Q}, \perp} /(\sqrt{\lambda} T)$ and $\operatorname{ImV}_{Q \bar{Q}, \|} /(\sqrt{\lambda} T)$ become more and more bigger. This behavior can also be seen from the right panel of fig.6, which shows $L_{\text {min }}$ versus $q$. One finds that as $q$ increases, $L_{\text {min }}$ increases, which indicates that the suppression becomes weaker. On the other hand, the results show that as $q$ increases, the differences between $L_{m i n, \perp}$ and $L_{\min , \|}$ widen, especially at large $\beta$. Also, at fixed $\beta$, the slope of $L_{m i n, \perp}$ is smaller than that of $L_{m i n, \|}$, which implies that the D-instanton density has smaller effects for the transverse case rather than parallel, differs from the velocity, which has stronger effects for the perpendicular case [24, 25].

\section{CONCLUSION AND DISCUSSION}

In this paper, we studied the imaginary potential of moving quarkonia in a D-instanton background, generated by a dilaton field, corresponding to D-instanton density contributions. The features of the background configuration are 

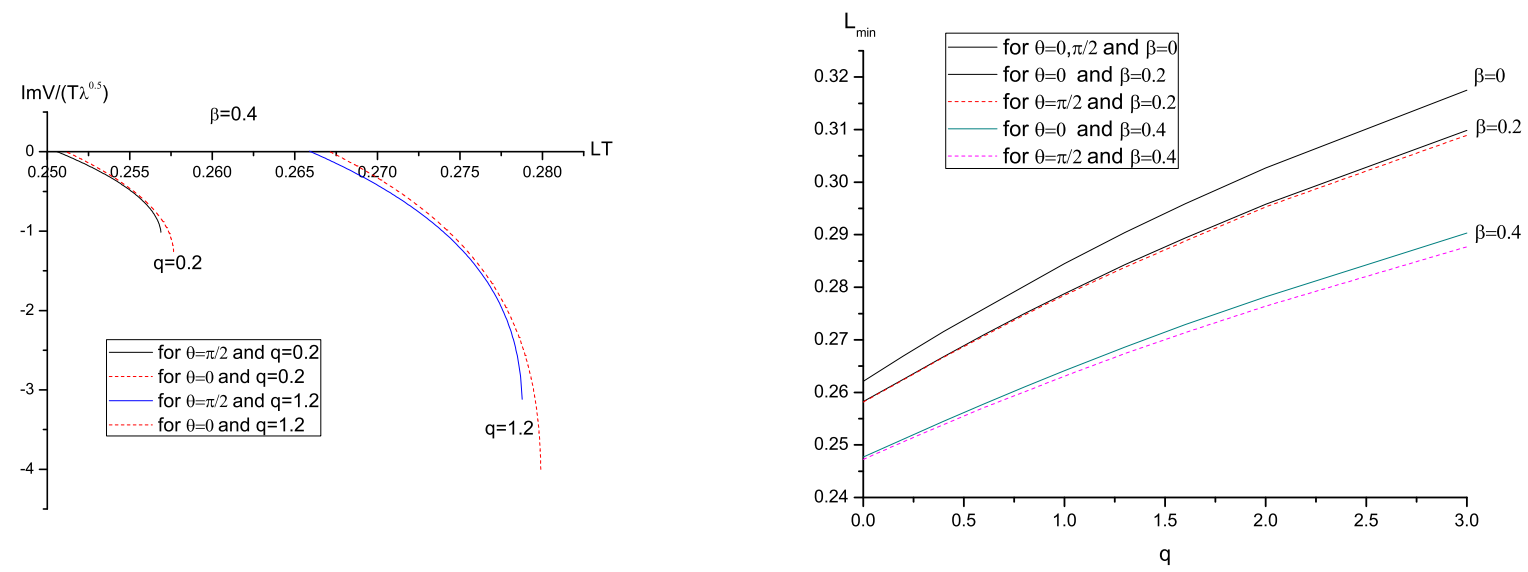

FIG. 6: Left: $\operatorname{Im} V /(\sqrt{\lambda} T)$ versus $L T$ for $\beta=0.4$. Right: $L T_{\min }$ versus $q$ for $\beta=0,0.2,0.4$, respectively.

similar to QCD at finite temperature. Thus, one expects the results obtained from this theory should shed qualitative insights into analogous questions in QCD.

To analyze the imaginary potential, we considered the quark anti-quark pair moving transverse and parallel to the plasma wind, respectively. For both cases, it is found that the D-instanton density increases the inter-distance and decreases the imaginary potential, reverse to the effect of the pair's velocity. Since the dissociation properties of quarkonia should be sensitive to the imaginary potential, and the larger the $\operatorname{ImV}_{Q Q}$, the stronger the suppression. Thus, one concludes that the presence of the D-instanton density makes the suppression weaker, reverse to the effect of the velocity. Furthermore, it is shown that the D-instanton density has stronger effects for the parallel case rather than perpendicular.

Finally, it would be interesting to mention that the entropic force has been recently argued to be responsible for melting heavy quarkonia [44, 45]. It is also of interest to study this quantity in the D-instanton background. We leave this for further study.

\section{ACKNOWLEDGMENTS}

This research is partly supported by the Ministry of Science and Technology of China (MSTC) under the 973 Project No. 2015CB856904(4). Z-q Zhang is supported by NSFC under Grant No. 11705166. G.C is supported by NSFC under Grant No. 11475149. D-f. Hou is partly supported by the NSFC under Grants Nos. 11735007, 11375070, 11521064 .

[1] J. Adams et al. [STAR Collaboration], Nucl. Phys. A 757, 102 (2005).

[2] K. Adcox et al. [PHENIX Collaboration], Nucl. Phys. A 757, 184 (2005).

[3] E. V. Shuryak, Nucl. Phys. A 750, 64 (2005).

[4] T. Matsui, H. Satz, Phys. Lett. B 178, 416 (1986).

[5] M. Laine, O.Philipsen, P. Romatschke and M. Tassler, JHEP 03 (2007) 054

[6] A. Beraudo, J.-P. Blaizot, and C. Ratti, Nucl. Phys. A806, 312 (2008).

[7] N. Brambilla, J. Ghiglieri, A. Vairo, and P. Petreczky, Phys. Rev. D 78, 014017 (2008).

[8] M. A. Escobedo, J.Phys.Conf.Ser. 503 (2014) 012026.

[9] N. Brambilla, M.A. Escobedo, J. Ghiglieri, J. Soto and A. Vairo, JHEP 09 (2007) 038

[10] A. Dumitru, Y. Guo, and M. Strickland, Phys.Rev. D79 (2009) 114003.

[11] M. Margotta, K. McCarty, C. McGahan, M. Strickland, and D. Y. Elorriaga, Phys.Rev. D 83 (2011) 105019.

[12] V. Chandra and V. Ravishankar, Nucl. Phys. A 848 (2010) 330.

[13] N. Haque, A.Bandyopadhyay, J.O. Andersen, M. G. Mustafa, M. Strickland and N. Su, JHEP 05 (2014) 027.

[14] U.W Heinz, R. Snellings, Annu. Rev. Nucl. Part. Sci. 63 (2013) 123-151.

[15] S. Ryu, J.-F. Paquet, C. Shen, G. S. Denicol, B. Schenke, S. Jeon and C. Gale, Phys. Rev. Lett. 115, 132301 (2015).

[16] J. M. Maldacena, Adv. Theor. Math. Phys. 2, 231 (1998). 
[17] S. S. Gubser, I. R. Klebanov and A. M. Polyakov, Phys. Lett. B428, 105 (1998).

[18] O. Aharony, S. S. Gubser, J. Maldacena, H. Ooguri and Y. Oz, Phys. Rept. 323, 183 (2000).

[19] J. Casalderrey-Solana, H. Liu, D. Mateos, K. Rajagopal, and U. A. Wiedemann, arXiv:1101.0618

[20] J. Noronha, A. Dumitru, Phys. Rev. Lett. 103 (2009) 152304.

[21] S. I. Finazzo, J. Noronha, JHEP 11 (2013) 042.

[22] K. B. Fadafan, D. Giataganas and H. Soltanpanahi, JHEP 11 (2013) 107

[23] S. I. Finazzo, J. Noronha, JHEP 01 (2015) 051.

[24] M. Ali-Akbari, D. Giataganas and Z. Rezaei, Phys. Rev. D 90 (2014) 086001.

[25] K. B. Fadafan, S. K. Tabatabaei, J. Phys. G: Nucl. Part. Phys. 43095001 (2016).

[26] Z.q. Zhang, D.f. Hou, and G. Chen, Phys. Lett. B 768, 180 (2017).

[27] N. R. F. Braga and L. F. Ferreira, Phys. Rev. D 94, 094019 (2016).

[28] J. Sadeghi and S. Tahery, JHEP 06 (2015) 204.

[29] J. L. Albacete, Y. V. Kovchegov, A. Taliotis, Phys. Rev. D 78 (2008) 115007.

[30] T. Hayata, K. Nawa, T. Hatsuda, Phys. Rev. D 87 (2013) 101901.

[31] L. Hong and A. A. Tseytlin, Nuc. Phys. B 553, 231 (1999).

[32] B. Gwak, M. Kim, B.-H. Lee, Y. Seo, and S.-J. Sin, Phys. Rev. D 86, 026010 (2012).

[33] K. Ghoroku, T. Sakaguchi, N. Uekusa, and M. Yahiro, Phys. Rev. D 71, 106002 (2005).

[34] Z.q. Zhang, D.f. Hou, and G. Chen, Eur. Phys. J. A 52357 (2016).

[35] G. W. Gibbons, M. B. Green and M. J. Perry, Phys. Lett. B 370, 37 (1996).

[36] A. Kehagias and K. Sfetsos, Phys. Lett. B 456, 22 (1999).

[37] K. Ghoroku and M. Yahiro, Phys. Lett. B 604, 235 (2004).

[38] J. M. Maldacena, Phys. Rev. Lett. 80, 4859 (1998).

[39] A. Brandhuber, N. Itzhaki, J. Sonnenschein and S. Yankielowicz, Phys. Lett. B 434, 36 (1998).

[40] S. -J. Rey, S. Theisen and J. -T. Yee, Nucl. Phys. B 527, 171 (1998).

[41] D. Bak, A. Karch, L. G. Yaffe, JHEP 0708 (2007) 049.

[42] H. Liu, K. Rajagopal and U. A. Wiedemann, Phys. Rev. Lett. 98, 182301 (2007).

[43] H. Liu, K. Rajagopal and U. A. Wiedemann, JHEP 0703, 066 (2007).

[44] D. E. Kharzeev, Phys. Rev. D 90, 074007 (2014).

[45] K. Hashimoto and D. E. Kharzeev, Phys. Rev. D 90, 125012 (2014). 